\title{
Searches for supersymmetric higgsinos with the ATLAS detector
}

\author{
Lorenzo Rossini* \\ On behalf of the ATLAS Collaboration \\ Dipartimento di Fisica, Università degli studi di Milano \\ INFN, Sezione di Milano \\ Milano, Italy \\ E-mail: lorenzo.rossini@mi.infn.it
}

\begin{abstract}
A search for supersymmetric partners of the Higgs and gauge bosons (charginos and neutralinos) is presented in this work, using the data from the ATLAS experiment at the LHC. Considerations on the naturalness of the Higgs boson mass suggest that the two lightest neutralinos might be a mix of the partners of the Higgs and could have a similar mass. Using this scenario as reference, the analysis searches for final states with pairs of electrons and muons with low transverse momentums coming from the decay of the second lightest neutralino into the lightest neutralino, high missing transverse momentum generated by neutrinos which are non detectable, and an energetic jet coming from QCD initial state radiation. Results are shown for an analysis obtained with 36 $\mathrm{fb}^{-1}$, and limits are set on the mass parameter of the higgsinos.
\end{abstract}

An Alpine LHC Physics Summit (ALPS2018)

15-20 April, 2018

Obergurgl, Austria

\footnotetext{
* Speaker.
} 


\section{Introduction}

Supersymmetry (SUSY) [1] predicts that for each Standard Model particle exists a new state that differ just by half a unit of spin. Similar to the Standard Model, the superparteners of the gauge bosons (the Wino and Bino) mix together to give mass eigenstates called charginos, $\tilde{\chi}_{1,2}^{ \pm}$, and neutralinos, $\tilde{\chi}_{1,2,3,4}^{0}$ (where the subscripts indicate increasing mass) or collectively electroweakinos. The lightest neutralino is supposed to be stable due to R-parity conservation. If the mass parameters of the Wino and Bino are large with respect to the Higgsino (the superpartner of the Higgs boson), the two lightest neutralinos and the lightest chargino are composed mainly of higgsinos and form a triplet of states with a mass close to the higgsino mass parameter $\mu$ [2]. The mass splitting between the three states is between few hundred $\mathrm{MeV}$ to a few tens of $\mathrm{GeV}$, depending on how much the higgsinos are important in the mixture, and therefore the decay products of the $\tilde{\chi}_{2}^{0}$ into $\tilde{\chi}_{1}^{0}$ are very soft.

Moreover, up until recently the only experimental constraint comes from the LEP experiments, almost 20 years ago. The analysis presented here [3] searches for this kind of signature using the data collected with the ATLAS [4] detector at the LHC [5].

\section{Signal signature}

The signal considered is the direct production of a pair of $\tilde{\chi}_{2}^{0}$ and $\tilde{\chi}_{1}^{0}$, or $\tilde{\chi}_{2}^{0}$ and $\tilde{\chi}_{1}^{ \pm}$, where the $\tilde{\chi}_{2}^{0}$ decays into a $\tilde{\chi}_{1}^{0}$ and (through a virtual $Z^{0}$ ) two opposite sign same flavour leptons. The signal is defined by the presence of large missing transverse momentum (denoted $E_{T}^{\text {miss }}$ ) due to the two neutralinos boosted against a very energetic jet from initial state radiation (ISR). This topology allows to trigger on the $E_{T}^{\text {miss }}$ and look at the two soft leptons from the decay.

The invariant mass $\left(m_{l l}\right)$ of the two leptons is bounded to the difference in mass of the two neutralinos, and its shape depends on the higgsino content. The main strategy of the analysis is therefore to use a binned likelihood fit in invariant mass in order to separate signal and background. In case of discovery, the shape of the $m_{l l}$ distribution could give information on the nature of the signal.

\section{Analysis strategy}

In order to enhance the sensitivity of the analysis a set of topological cuts (Signal Region) is applied to the events collected by the ATLAS detector during 2015 and 2016. They are described in table 1.

These cuts are optimized to reduce the main sources of background: non prompt lepton (fakes) and instrumental $E_{T}^{\text {miss }}, Z \rightarrow \tau \tau$, diboson ( $W W, W Z, Z Z$ ) production, and top quark pair production. The main background Monte Carlo processes are normalized to data in Control Regions. These regions are defined in order to be as close as possible to the Signal Region but with low contamination from the signal. Validation Regions are also defined in order to check the correct modelling of the Monte Carlo and the fakes, which instead are estimated with a data driven method, called Fake Factor. 
Table 1: Summary of event selection criteria.

\begin{tabular}{ll}
\hline Variable & Common requirement \\
\hline Number of leptons & $=2$ \\
Lepton charge and flavor & $e^{+} e^{-}$or $\mu^{+} \mu^{-}$ \\
Leading lepton $p_{T}^{\ell_{1}}$ & $>5(5) \mathrm{GeV}$ for electron (muon) \\
Subleading lepton $p_{T}^{\ell_{2}}$ & $>4.5(4) \mathrm{GeV}$ for electron (muon) \\
$m_{l l}$ & $\in[1,60] \mathrm{GeV}$ excluding $[3.0,3.2] \mathrm{GeV}$ \\
$E_{T}^{\text {miss }}$ & $>200 \mathrm{GeV}$ \\
Number of jets & $\geq 1$ \\
Leading jet $p_{T}$ & $>100 \mathrm{GeV}$ \\
$\Delta \phi\left(j_{1}, \mathbf{p}_{\mathrm{T}}^{\text {miss }}\right)$ & $>2.0$ \\
min $\Delta \phi\left(\right.$ all jets, $\left.\mathbf{p}_{\mathrm{T}}^{\text {miss }}\right)$ & $>0.4$ \\
Number of $b$-tagged jets & $=0$ \\
$m_{\tau \tau}$ & $<0$ or $>160 \mathrm{GeV}$ \\
$\Delta R_{\ell \ell}$ & $>0.05 \&<2$ \\
$m_{\mathrm{T}}^{\ell_{1}}$ & $<70 \mathrm{GeV}$ \\
$E_{T}^{\text {miss }} / H_{\mathrm{T}}^{\text {leptons }}$ & $>\max \left(5,15-2 \frac{m_{l l}}{1 \mathrm{GeV}}\right)$ \\
\hline
\end{tabular}

\section{Results}

Data are in good agreement with the background estimation in the Validation Regions and therefore it is possible to look for an eventual excess in the Signal Region. No statistical significant excess has been observed and therefore only limits on the particles masses have been set. The exclusion limits at $95 \%$ Confidence level in the hypothesis of electroweakinos composed of mainly higgsinos is show as the blue contour in figure 1. This are the first LHC limits on this hypothesis. The LEP limits [6] are also shown in the grey area, while the orange region is the limits from another ATLAS analysis using the "disappearing track" and the pure Higgsinos hypothesis from [7].

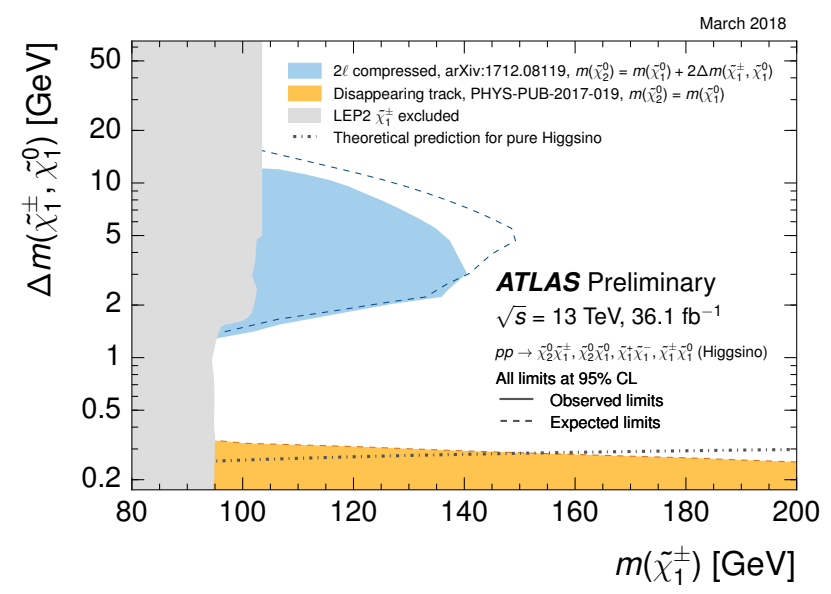

Figure 1: Exclusion limits at 95\% CL for higgsino pair production. The production cross-section is for pure higgsinos (from [8]) 


\section{References}

[1] Yu. A. Golfand and E. P. Likhtman, Extension of the Algebra of Poincare Group Generators and Violation of p Invariance, JETP Lett. 13 (1971) 323, [Pisma Zh. Eksp. Teor. Fiz. 13 (1971) 452].

[2] M. Papucci, J. T. Ruderman, and A. Weiler, Natural SUSY endures, JHEP 09 (2012) 035 arXiv: 1110.6926 [hep-ph].

[3] ATLAS Collaboration, Search for electroweak production of supersymmetric states in scenarios with compressed mass spectra at $\sqrt{s}=13 \mathrm{TeV}$ with the ATLAS detector, Phys. Rev. D 97 (2018) 052010

[4] ATLAS Collaboration, The ATLAS Experiment at the CERN Large Hadron Collider, JINST 3 (2008) S08003.

[5] L. Evans and P. Bryant, LHC Machine, JINST 3 (2008) S08001.

[6] ALEPH, DELPHI, L3, OPAL Experiments, Combined LEP Chargino Results, up to $208 \mathrm{GeV}$ for low DM, LEPSUSYWG/02-04.1, 2002, url:

http://lepsusy.web.cern.ch/lepsusy/www/inoslowdmsummer02/charginolowdm_pub.html

[7] ATLAS Collaboration, Search for long-lived charginos based on a disappearing-track signature in $p p$ collisions at $\sqrt{s}=13 \mathrm{TeV}$ with the ATLAS detector, JHEP 1806 (2018) 022

[8] https://atlas.web.cern.ch/Atlas/GROUPS/PHYSICS/CombinedSummaryPlots/SUSY/ 